\title{
Analysis of the finiteness for the first collision time between two randomly moving particles
}

Alaa A. Alzulaibani(D)

\author{
Correspondence: azulaibani@ \\ taibahu.edu.sa \\ Mathematics and Statistics \\ Department, College of Science, \\ Taibah University, Yanbu, Kingdom \\ of Saudi Arabia
}

\begin{abstract}
The finiteness of the collision time between two different randomly moving particles is presented by providing more useful analysis that gives stronger and finite moment. The triangular arrays and the uniform integrability conditions of the all probable positions non-stationary random sequence are used. In addition, an important property of Marcinkiewicz laws of large numbers and Hoffman-Jorgensen inequality are presented in this analysis. All of them are deriving to provide the sufficient conditions that give more stronger moments of the first meeting time in the probability space.
\end{abstract}

Keywords: Non-stationary random sequence, First meeting time, Triangular arrays Mathematics subject classification: $46 \mathrm{~N} 55,60 \mathrm{G} 40,76 \mathrm{M} 35,46 \mathrm{~S} 50$

\section{Highlights}

- More useful analysis is presented to provide the finiteness of the first meeting time between two randomly moving particles.

- All probable positions of the first collision time are considered as a random sequence defined on the probability space.

- The triangular arrays and the uniform integrability conditions of the all probable positions are used to obtain the sufficient conditions which give the stronger moments.

- An important and useful property of Marcinkiewicz laws of large numbers is presented.

- A new and useful result is obtained by using Hoffman-Jorgensen inequality.

\section{Introduction}

The particles move in the fluid with one of the famous stochastic processes such as Levy process and Brownian motion. Physicists are concerned in studying the physical properties of the particles movement in the reactive medium. The important is studying the finiteness of the collision time expected value between different kinds of

(c) The Author(s). 2020 Open Access This article is licensed under a Creative Commons Attribution 4.0 International License, which permits use, sharing, adaptation, distribution and reproduction in any medium or format, as long as you give appropriate credit to the original author(s) and the source, provide a link to the Creative Commons licence, and indicate if changes were made. The images or other third party material in this article are included in the article's Creative Commons licence, unless indicated otherwise in a credit line to the material. If material is not included in the article's Creative Commons licence and your intended use is not permitted by statutory regulation or exceeds the permitted use, you will need to obtain permission directly from the copyright holder. To view a copy of this licence, visit http://creativecommons.org/licenses/by/4.0/. 
randomly moving particles within this medium. In the case of linear flows in the fluid, El-Hadidy [1] and Alzulaibani [2] provided advanced analysis to get the sufficient conditions of this finiteness. They showed that the random sequence of all random variables of all probable positions of the first collision time is uniformly integerable function and has a triangular array. This will give a stronger moment of the first collision time expected value.

In this work, more useful analysis is derived to present the finiteness of expected value of the first collision time (that was provided in all models studied in El-Hadidy et al. [3, 4]). In addition, one can use our idea to present more suitable analysis for different $n$-dimensional models, for moving particles (targets) as studied in El-Hadidy et al. [5-15]. In these works, the authors get the first moment only, which is not useful for the sufficient finiteness of this expected value. In the case of the randomly located target, El-Hadidy et al. [16-21] presented statistical and analytical studies to maximize the probability of the target detection or minimize the expected value of the searching time. Here, let $Y_{1}, Y_{2}, \ldots, Y_{m}$ be a finite number of all probable positions of the first collision time defined on a given probability space $(\Omega, \boldsymbol{\aleph}, P)$ and they are independent identically distributed random variables (i.i.d.r.vs). In this probability space, $\Omega$ presents all first meeting probable positions, $\boldsymbol{N}$ is the $\sigma$ - algebra that provides all algebraic operations at time $t \in R^{+}$and $P$ is the probability measure for these operations. In addition, let the set of all linear paths of the randomly moving particle (moves with stochastic process $\left.s_{1}(t)\right)$ in the reactive medium be $\beta(t)$. The other particle moves with another stochastic process $s_{2}(t)$. Define $\beta(t):[0, \infty) \rightarrow[0, \infty)$ as a class of all convex functions such that $\beta(2 t)=c \beta(t), c>0$. El-Hadidy [1] showed that the first moment of the first collision time expected value is finite if $W_{1}, W_{2}, \ldots, W_{m}$ is uniformly integrable as in the following Lemma 1 and Theorem 1.

Lemma 1. The set of all random variables $W_{1}, W_{2}, \ldots, W_{m}$ is uniformly integrable iff $\operatorname{Sup}_{m} E\left[\beta\left(\left|W_{m}\right|\right)\right]<\infty$, for some $\hat{\beta}\left(\left|W_{m}\right|\right) \in \beta\left(\left|W_{m}\right|\right)$.

Theorem 1. If $\left\{W_{k}\right\}_{k \in N}, N=\{1,2, \ldots\}$ (sequence of all non stationary and i.i.d.r.vs), $\beta(t):[0, \infty) \rightarrow[0, \infty)$ (be a non-decreasing function), $\operatorname{Sup}_{m} E\left[\beta\left(\left|W_{m}\right|\right)\right]<\infty$ and $t_{\eta}=$ $\inf \left\{t \in[0, \infty] ; P\left(W_{1}>t, W_{2}>t, \ldots, W_{\zeta}>t\right) \leq \frac{t}{c^{2}}-\alpha_{q}\right\}$ for $\zeta>q \geq 1, \eta \in(0,1)$, then

$$
E\left[\beta\left(W_{m}\right)\right] \leq c^{2}\left(\prod_{k=1}^{\zeta} E\left[\beta_{k}\left(q M_{k}\right)\right]+\left(\beta_{q}+\eta\right) \prod_{k=1}^{\zeta} E\left[\beta_{k}\left(W_{k}\right)\right]+\beta\left(t_{\eta}\right)\right) .
$$

Alzulaibani [2] showed that the triangular arrays of $\left\{W_{k}\right\}_{k \in N}$ on $(\Omega, \boldsymbol{N}, P)$, as in the following Theorem 2 is sufficient to get more stronger moments for the finiteness of the first meeting time.

Theorem 2. If $\left\{W_{k}, 1 \leq k \leq k_{n}, n \geq 1\right\}$ is a triangular array, $V_{n k}=\sum_{i=1}^{k} r_{i} W_{n i}, V_{n}=V_{n k_{n}}$ $r_{i}$ is the rth moment degree of the variable $W_{n i}$ and $\rho_{n}^{2}=\sum_{k=1}^{k_{n}} W_{n k}^{2}$, then: $\left\{V_{n}^{2}\right\}_{n \in N}$ is uniformly integrable iff $\left\{\rho_{n}^{2}\right\}_{n \in N}$ is uniformly integrable.

This paper is organized as follows: Section 2 presents collection of different results which give more stronger moments. In this section, some Theorems, Lemmas, and Proposition are presented by using the Marcinkiewicz laws of large numbers and 
Hoffman-Jorgensen inequality. Finally, the concluding remarks about these results and the future works are provided.

\section{Analysis of the finiteness}

Let all probable positions of the first collision time between two randomly moving particles $\left\{W, W_{1}, W_{2}, \ldots, W_{k}, \ldots\right\}$ be a (not necessarily stationary) random sequence defined on a $(\Omega, \boldsymbol{N}, P)$ and $\boldsymbol{\aleph}_{k}^{m}$ be the $\sigma$ - field generated by $\left\{W_{k}, W_{k+1}, \ldots, W_{m}, m \in N\right\}$. Also, let the coefficient $\phi_{n}$ defined by: $\phi_{n}=\operatorname{Sup}_{k \in N} \operatorname{Sup}\left\{P(B \mid A)-P(B) ; P(A)>0, A \in \boldsymbol{N}_{1}^{k}, B \in \boldsymbol{N}_{n+k}^{\infty}\right.$ \} ; if $\mathrm{A}$ and $\mathrm{B}$ are two events in $(\Omega, \boldsymbol{N}, P)$, then let $\psi_{n}=\operatorname{Sup}_{k \in N} \operatorname{Sup}\left\{\left|\frac{P(A \cap B)}{P(A) P(B)}-1\right| ; P(A) P\right.$ $\left.(B)>0, A \in \boldsymbol{N} F_{1}^{k}, B \in \boldsymbol{\aleph}_{n+k}^{\infty}\right\} ; \quad \psi_{n}^{*}=\operatorname{Sup}_{k \in N} \operatorname{Sup}\left\{\frac{P(A \cap B)}{P(A) P(B)} ; P(A) P(B)>0, A \in \boldsymbol{\aleph}_{1}^{k}, B \in \boldsymbol{\aleph}_{n+k}^{\infty}\right\} ;$ $\psi_{n}^{\prime}=\inf _{k \in N} \inf \left\{\frac{P(A \cap B)}{P(A) P(B)} ; P(A) P(B)>0, A \in \boldsymbol{\aleph}_{1}^{k}, B \in \boldsymbol{\aleph}_{n+k}^{\infty}\right\}$; which are the coefficients of dependence and they are stronger than $\phi_{n}$. Also, let $M_{n}=\underset{1 \leq k \leq n}{\max }\left|W_{k}\right|$.

Proposition 1. Assuming that $n>m \geq 1$ and $\phi_{m}<1$, for $V_{k} \in R^{+}$, one can obtain,

$$
P\left[\max _{1 \leq k \leq n-m+1}\left|V_{k}\right|>t\right] \leq \frac{3}{1-\phi_{m}} \max _{m \leq k \leq n} P\left[\left|V_{k}\right|+(m-1) M_{n}>\frac{t}{3}\right] .
$$

If $\varphi_{m<\frac{1}{2}}$ and $\tau\left(V_{n}-V_{k}\right)$ are symmetric for $\mathrm{n}>\mathrm{k} \geq 1$ then,

$$
P\left[\left|V_{n}\right|+(m-1) \max _{1 \leq i \leq n}\left|X_{i}\right|>t\right] \geq\left(\frac{1}{2}-\varphi \mathrm{m}\right) P\left[\max _{1 \leq k \leq n-m+1}\left|V_{k}\right|>t\right] .
$$

Proof: Assuming that $n>m, C_{1}=\left\{V_{1}>t+v\right\}$ and for $1<k \leq n, v>0$, one can get,

$$
C_{k}=\left\{V_{1} \leq v+t, \ldots, V_{k-1} \leq v+t, V_{k}>v+t\right\} .
$$

Also, let $V_{k}^{m}=\left\{\begin{array}{c}0, \text { if } k \geq m \\ \sum_{s=k+1}^{k+m-1} W_{s}, \text { otherwise }\end{array}\right.$ As in Kolmogorov [22], for $1 \leq k \leq n-m+1$, one can obtain $\mathcal{C}_{k} \cap\left\{=k+V_{n}+V_{k+m-1} \leq v\right\} \subseteq C_{k} \cap\left\{V_{n}-V_{k}^{m}>t\right\}$ and $\sum_{k=1}^{n} C_{k}=\left\{\max _{1 \leq k \leq n} V_{k}\right.$ $>v+t\}$. Thus, $\quad P\left[V_{n}+(m-1) \max _{1 \leq i \leq n}\left|W_{i}\right|>t\right] \geq P\left[V_{n}+(m-1) \max _{1 \leq i \leq n}\left|W_{i}\right|>t, \max _{1 \leq k \leq n} V_{k}\right.$ $\left.+(m-1) M_{n}>v+t\right]$

$$
\begin{aligned}
& \geq \sum_{k=1}^{n} P\left[C_{k}, V_{n}+(m-1) M_{n}>t\right] \geq \sum_{k=1}^{n-m+1} P\left[C_{k}, S V_{n}-V_{k}^{m}>t\right] \\
& \geq \sum_{k=1}^{n-m+1} \quad P\left[C_{k},-V_{n}+V_{k+m-1} \leq v\right] \geq\left(\min _{1 \leq k \leq n-m} P\left[-V_{n}+V_{k+m-1} \leq v\right]-\phi_{m}\right) \\
& \left.\max _{1 \leq k \leq n-m+1} V_{k}>v+t\right] .
\end{aligned}
$$

Also, according to Lin and Lu [23], for $n>k \geq 1$, if $\mathcal{L}\left(V_{n}-V_{k}\right)$ are symmetric, then $P$ [

$$
\begin{aligned}
\left.V_{n}+(m-1) \max _{1 \leq i \leq n}\left|W_{i}\right|>t\right] & \geq\left(\frac{1}{2}-\varphi_{m}\right) P\left[\max _{1 \leq k \leq n-m+1} V_{k}>t\right] \text {.Since, } \\
& P\left[\max _{1 \leq k \leq n}\left|V_{k}\right|>t\right] \leq P\left[\max _{1 \leq k \leq n} V_{k}>t\right]+P\left[\max _{1 \leq k \leq n}\left(-V_{k}\right)>\right],
\end{aligned}
$$

then by using (4) and (5), and as in Skorokhod [24, 25], one can get $P\left[\left|V_{n}\right|+(m-1)\right.$ $\left.\max _{1 \leq i \leq n}\left|W_{i}\right|>t\right]$

$$
\geq\left(\min _{1 \leq k \leq n-m} P\left[\left|V_{n}-V_{k+m-1}\right| \leq v\right]-\phi_{m}\right) P\left[\max _{1 \leq k \leq n-m+1}\left|V_{k}\right|>v+t\right]
$$


Consequently,

$$
\begin{aligned}
P\left[\left|V_{n}\right|+(m-1)\right. & \left.\max _{1 \leq i \leq n}\left|W_{i}\right|>t\right] \\
& \geq\left(2 \min _{m \leq k \leq n} P\left[\left|V_{k}\right| \leq \frac{v}{2}\right]-\varphi_{m}\right) P\left[\max _{1 \leq k \leq n-m+1}\left|V_{k}\right|>v+t\right] .
\end{aligned}
$$

Let for any $t$, their exists $\max _{m \leq k \leq n} P\left[\left|V_{k}\right|>\frac{t}{3}\right] \leq \frac{1-\varphi_{m}}{3}$. If one replace $s$ by $\frac{2 t}{3}$ and $t$ by $\frac{t}{3}$, then he get,

$$
\begin{aligned}
\mathrm{P}\left[\max _{1 \leq k \leq n-m+1}\left|V_{k}\right|>t\right] & \leq \frac{P\left[\left|V_{n}\right|+(m-1) \max _{1 \leq i \leq n}\left|W_{i}\right|>\frac{t}{3}\right]}{1-\frac{2\left(1-\varphi_{m}\right)}{3}-\varphi_{m}} \\
& =\frac{3}{1-\varphi_{m}} P\left[\left|V_{n}\right|+(m-1) \max _{1 \leq i \leq n}\left|X_{i}\right|>\frac{t}{3}\right]
\end{aligned}
$$

It is clear that, for $\mathrm{n}>\mathrm{k} \geq 1$, if $\mathcal{L}\left(V_{n}-V_{k}\right)$ are symmetric and $\psi_{m}^{\prime}>0$, then for $n>$ $m \geq 1$, one can get,

$$
2 P\left[\left|V_{n}\right|+(m+1) \max _{1 \leq i \leq n}\left|W_{i}\right|>t\right] \geq \psi_{m}^{\prime} P\left[\max _{1 \leq k \leq n-m+1}\left|V_{k}\right|>t\right] .
$$

By using the above Proposition 1, Szewczak [26] presented the generalization of the Hoffman-Jorgensen inequality [27] as in the following Proposition 2.

Proposition 2. (Szewczak [15]) For $v>0, t>0, u>0$, if $m \in \mathbb{N}, n>m$, then one can get,

$$
\begin{array}{r}
P\left[\max _{1 \leq k \leq n}\left|V_{k}\right|>v+t+u\right] \leq P\left[\max _{1 \leq i \leq n}\left|W_{i}\right|>u\right] \\
+\left(\varphi_{m}+P\left[\max _{\substack{k+m \leq j \leq n \\
1 \leq k \leq n-m}}\left|\sum_{s=k+m}^{j} W_{s}\right|>t\right]\right) P\left[\max _{1 \leq k \leq n-m}\left|V_{k}\right|>v\right] .
\end{array}
$$

Theorem 3. If $\left\{W_{k}\right\}_{k \in N}, N=\{1,2, \ldots\}$ be a non-stationary random sequence, $M_{n}$ $=\sup _{1 \leq k \leq n}\left|\sum_{k=1}^{n} W_{k}\right|$ and if $E\left[\left|X_{1}\right|^{p}\right]<\infty, p>0$, then for $v>0, \alpha>0, n>m$,

$$
\begin{aligned}
& 2^{-p}(1+v)^{-p} E\left[\max _{1 \leq k \leq n}\left|V_{k}\right|^{p} I\left(\max _{1 \leq k \leq n}\left|V_{k}\right|^{p}>\alpha 2^{p}(1+v)^{p}\right)\right] \\
& \leq \mathfrak{p}_{m}^{n}(v \sqrt[p]{\alpha}) E\left[\max _{i \leq k \leq n}\left|V_{k}\right|^{p} I\left(\max _{1 \leq k \leq n}\left|V_{k}\right|^{p}>\alpha\right)\right]+m^{p} E\left[M_{n}^{p} I_{\left(m^{p} M_{n}^{p}>\alpha\right)}\right],
\end{aligned}
$$

where $I_{(.)}$is the indicator function and $\mathfrak{p}_{m}^{n}(u)=\phi_{m}+P\left[\max _{m \leq k \leq n}\left|V_{k}\right|>u\right]$. Proof: From Proposition 2 if $n>m \geq 1$, then one can get,

$$
P\left[\max _{1 \leq i \leq n}\left|V_{k}\right|>v+2 t+u\right] \leq P\left[m . \max _{1 \leq i \leq n}\left|X_{i}\right|>u\right]+\psi_{m}^{*} P\left[\max _{m \leq k \leq n}\left|V_{k}\right|>t\right] P\left[\max _{1 \leq k \leq n-m}\left|V_{k}\right|>v\right] .
$$

Now, let $Z_{n}=\max _{1 \leq k \leq n}\left|V_{k}\right|$ this implies that,

$$
P\left[Z_{n}>2(t+v)\right] \leq \mathfrak{p}_{m}^{n}(s) P\left[Z_{n}>t\right]+P\left[m M_{n}>t\right]
$$

Thus, if I put $v=v t$, then I get,

$$
P\left[Z_{n}>2(1+v) t\right] \leq \mathfrak{p}_{m}^{n}(v t) P\left[Z_{n}>t\right]+P\left[m M_{n}>t\right] .
$$

And, let $Z$ be a positive random variable, then one can get, 


$$
E\left[Z I_{(Z>\alpha)}\right]=\alpha P[Z>\alpha\}+\int_{\alpha}^{\infty} P[Z>u] d u
$$

Consequently,

$$
\begin{aligned}
& E\left[Z_{n}^{p} I_{\left(Z_{n}^{p}>2^{p}(1+v)^{p}\right.}\right] \leq 2^{p}(1+v)^{p} \alpha P\left[Z_{n}^{p}>2^{p}(1+v)^{p} \alpha\right]+\int_{2 p(1+v)^{p} \alpha}^{\infty} P\left[Z_{n}^{p}>t\right] d t \\
& \leq 2^{p}(1+v)^{p} \alpha\left(\mathfrak{p}_{m}^{n}(v \sqrt[p]{\alpha}) P\left[Z_{n}>\sqrt[p]{\alpha}\right]+P\left[m M_{n}>\sqrt[p]{\alpha}\right]\right) \\
&+2^{p}(1+v)^{p} \int_{\alpha}^{\infty} P\left[Z_{n}>2(1+v) \sqrt[p]{t}\right] d t \\
& \leq 2^{p}(1+v)^{p} \alpha\left(\mathfrak{p}_{m}^{n}(v \sqrt[p]{\alpha}) P\left[Z_{n}>\sqrt[p]{\alpha}\right]+P\left[m M_{n}>\sqrt[p]{\alpha}\right]\right) \\
&+2^{p}(1+v)^{p} \int_{\alpha}^{\infty} P_{m}^{n}(v \sqrt[p]{t}) P\left[Z_{n}>\sqrt[p]{t}\right] d t \\
&+2^{p}(1+v)^{p} \int_{\alpha}^{\infty} P\left[m M_{n}>\sqrt[p]{t}\right] d t \\
& \leq 2^{p}(1+v)^{p} \mathfrak{p}_{m}^{n}(v \sqrt[p]{\alpha})\left(\alpha P\left[Z_{n}>\sqrt[p]{\alpha}\right]+\int_{\alpha}^{\infty} P\left[Z_{n}>\sqrt[p]{t}\right]\right) d t \\
&+2^{p}(1+v)^{p}\left(\alpha P\left[m M_{n}>\sqrt[p]{\alpha}\right]+\int_{\alpha}^{\infty} P\left[m M_{n}>\sqrt[p]{t}\right]\right) d t \\
&=2^{p}(1+v)^{p}\left(\mathfrak{p}_{m}^{n}(v \sqrt[p]{\alpha}) E\left[Z_{n}^{p} I\left(Z_{n}^{p}>\alpha\right)\right]+m^{p} E\left[M_{n}^{p} I\left(m^{p} M_{n}^{p}>\alpha\right)\right]\right) .
\end{aligned}
$$

If $E\left[\left|X_{1}\right|^{p}\right]<\infty, p>0, n>m \geq 1, \tau \in(0,1)$ and $t_{\tau}=\inf \left\{t>0 ; \phi_{m}+P\left[\max _{m \leq k \leq n}\left|V_{k}\right|>t\right]\right.$ $\left.\leq 4^{-p} \tau\right\}$, then one can obtain the same result as in Szewczak [26],

$$
E\left[\max _{m \leq k \leq n}\left|V_{k}\right|^{p}\right] \leq \frac{4^{p}}{1-\tau}\left(m^{p} E\left[\max _{1 \leq i \leq n}\left|W_{i}\right|^{p}\right]+t_{\tau}^{p}\right) .
$$

As in Szewczak [26] (Proposition 12), the strictly stationary sequence $\left\{W_{k}\right\}_{k \in N}$ of all probable positions of the first meeting time satisfies:

$$
\left(1-\phi_{m}\right) P\left[M_{[n / m}^{*}>w\right] \leq P\left[M_{n}>w\right] \leq m\left(1-\phi_{m}\right) P\left[M_{[n / m]+1}^{*}>w\right],
$$

where $\phi_{m}<1$ and $y \geq 0, n \geq m \geq 1$. That is useful with the above propositions to prove the following Theorem 4 which provides the maxima of a strictly stationary sequence $\left\{W_{k}\right\}_{k \in N}$. This gives more stronger moments for the finiteness of the first collision time at any random position $w$ in the reactive medium.

Theorem 4. For the strictly stationary random sequence $\left\{W_{k}\right\}_{k \in N}$, if $\varphi_{m}<1, w_{\vartheta}=$ $\sup \left\{x ; P\left[\left|W_{1}\right|\right]<1\right\}>0$ and $q>0, w_{\vartheta}>w>0$, then

$$
\left(1-\varphi_{m}\right) \frac{[n / m] E\left[\left|W_{1}\right|^{q} I_{\left[\left|w_{1}\right|>w\right]}\right]}{1+[n / m] P\left[\left|W_{1}\right|>w\right]} \leq E\left[M_{n}^{q}\right], \quad n \geq m .
$$

The direct consequence of Lemmas and Theorems in this paper, are useful to get the finiteness of the first meeting time between the randomly moving particles in the reactive medium.

\section{Concluding remarks}

The main contributions of this paper can be summarized as follows: 
1. More useful analysis that shows the finiteness of the collision time between two different randomly moving particles has been discussed.

2. The triangular arrays and uniform integrability conditions of the non-stationary random sequence of all probable positions are used.

3. The sufficient conditions that give more stronger moments of the collision time in the probability space has been presented.

4. New results obtained here are more useful and general than the results in ElHadidy [1] and Alzulaibani [2].

5. In the future research, one can apply more advanced analysis by studying these sufficient conditions for the $n$-dimensional stochastic particle motion in the fluid.

Acknowledgements

The author gratefully acknowledges the anonymous referees for their insightful and constructive comments and suggestions.

Author's contributions

The author has made each part of this paper. He read and approved the final manuscript.

Funding

Not applicable.

Availability of data and materials

Not applicable

Competing interests

The author declares that he has no competing interests.

Received: 19 January 2020 Accepted: 17 May 2020

Published online: 01 July 2020

\section{References}

1. El-Hadidy, H.: Studying the finiteness of the first meeting time between Levy flight jump and Brownian particles in the fluid reactive anomalous transport, Modern Physics Letters B, Vol. 33, No. 22 (2019) 1950256 (8 pages).

2. Alzulaibani, $A$ :: On the finiteness of the first meeting time between a Gaussian jump and a Brownian particles in the fluid. Modern Physics Letters B, In Press. (2020). https://doi.org/10.1142/S0217984920501390

3. El-Hadidy, M., Abou-Gabal, H.: Coordinated search for a random walk target motion, Fluctuation and Noise Letters, Vol. 17, No. 1 (2018), 1850002 (11 pages).

4. El-Hadidy, M., Alzulaibani, A.: Cooperative search model for finding a Brownian target on the real line. Journal of Taibah University for Science. 13(1), 177-183 (2019)

5. El-Hadidy, M.: Generalised linear search plan for a D-dimensional random walk target. International Journal of Mathematics in Operational Research. 15(2), 211-241 (2019)

6. El-Hadidy, M.: Existence of finite parabolic spiral search plan for a Brownian target'. International Journal of Operational Research. 31(1), 368-383 (2018)

7. El-Hadidy, M.: Fuzzy optimal search plan for N-dimensional randomly moving target, International Journal of Computational Methods, 13 (6)(2016) 1650038 (38 pages).

8. El-Hadidy, M.: Searching for a d-dimensional Brownian target with multiple sensors. International Journal Mathematics in Operational Research. 9(3), 279-301 (2016)

9. Mohamed, A., Kassem, M., El-Hadidy, M.: 'Multiplicative linear search for a Brownian target motion. Appli. Mathematical Model. 35(9), 4127-4139 (2011)

10. El-Hadidy, M., Alzulaibani, A.: Existence of a finite multiplicative search plan with random distances and velocities to find a D-dimensional Brownian target. Journal of Taibah University for Science. 13(1), 1035-1043 (2019)

11. Alzulaibani, A., El-hadidy, M.: Study on the finiteness of the first meeting time between N-dimensional Gaussian jump and Brownian diffusion particles in the fluid, International Journal of Modern Physics B, Vol. 33, No. 0 (2019) 1950334 (22 pages). DOI: https://doi.org/10.1142/S021797921950334X.

12. El-hadidy, M.: Existence of cooperative search technique to find a Brownian target. Journal of the Egyptian Mathematical Society. 28(1), 1-12 (2020). https://doi.org/10.1186/s42787-019-0054-5

13. El-Hadidy M., Alfreedi A., and Alzulaibani A.: Optimal multiplicative generalized coordinated search technique to find a D-dimensional random walker, Int. J. of Operational Research, (2019), In Press. http://www.inderscience.com/info/ ingeneral/forthcoming.php?jcode=ijor. DOl: https://doi.org/10.1504/IJOR.2021.10024709.

14. El-Hadidy, M., Abou-Gabal, H.: Searching for the random walking microorganism cells. International Journal of Biomathematics. 12(6), 1950064 (2019) (12 pages)

15. El-Hadidy, M.: On the existence of a finite linear search plan with random distances and velocities for a one-dimensional Brownian target. International Journal of Operational Research. 37(2), 245-258 (2020)

16. El-Hadidy, M., Alfreedi, A.: Minimizing the expected search time of finding the hidden object by maximizing the discount effort reward search. Journal of Taibah University for Science. 14(1), 479-487 (2020) 
17. Alfreedi, A., El-Hadidy, M.: On optimal coordinated search technique to find a randomly located target. Statistics, Optimization \& Information Computing. 7(4), 854-863 (2019)

18. El-Hadidy, M., Teamah, A., El-Bagoury, A.: 3-dimensional coordinated search technique for a randomly located target. International Journal of Computing Science and Mathematics. 9(3), 258-272 (2018)

19. El-Hadidy, M.: On maximum discounted effort reward search problem. Asia-Pacific Journal of Operational Research. 33(03), 1650019 (2016)

20. Teamah, A., Kassem, M., El-Hadidy, M.: M-states search problem for a lost target with multiple sensors. International Journal of Mathematics in Operational Research. 10(1), 104-135 (2017)

21. El-Hadidy M., Fakharany M.: Optimal 3-dimensional search model to find the underwater randomly hidden target, International Journal of Mathematics in Operational Research, In Press (2020). https://www.inderscience.com/info/ ingeneral/forthcoming.php?jcode=ijmor

22. Kolmogorov, A.N.: Uber die Summen durch den Zufall bestimmter unabh"angiger Grosen. Math. Ann. 99, 309-319 (1928)

23. Lin, Z.Y., Lu, C.R., Limit theory for mixing dependent random variables, Mathematics and its Application v. 378, Science Press (Beijing, 1996).

24. Skorokhod, A.V.: Limit theorems for stochastic processes with independent increments. Theory Probab. Appl. 2, 138-171 (1957)

25. Skorokhod, A.V:: Random processes with independent increments, Nauka (Moscow, 1964) (in Russian)

26. Szewczak, Z.S.: Marcinkiewicz laws with infinite moments. Acta Math. Hungar. 127, 64-84 (2010)

27. Hoffmann-Jorgensen, J: Sums of independent Banach space valued random variables. Studia Math. 52, 159-186 (1974)

\section{Publisher's Note}

Springer Nature remains neutral with regard to jurisdictional claims in published maps and institutional affiliations.

\section{Submit your manuscript to a SpringerOpen ${ }^{\circ}$ journal and benefit from:}

- Convenient online submission

- Rigorous peer review

- Open access: articles freely available online

- High visibility within the field

- Retaining the copyright to your article 